CAHIERS DE

NARRATOLOGIE

\section{Cahiers de Narratologie}

Analyse et théorie narratives

31 Bis | 2017

Espace du récit, récit de l'espace en contexte germanique

\title{
Orient imaginé/Orient vécu chez les Juifs germanophones (yekkes) en Palestine/Israël
}

\section{Patrick Farges}

\section{(2) OpenEdition \\ Journals}

Electronic version

URL: http://journals.openedition.org/narratologie/7687

DOI: $10.4000 /$ narratologie. 7687

ISSN: 1765-307X

Publisher

LIRCES

Electronic reference

Patrick Farges, «Orient imaginé/Orient vécu chez les Juifs germanophones (yekkes) en Palestine/ Israël », Cahiers de Narratologie [Online], 31 Bis | 2017, Online since 26 June 2017, connection on 15 November 2019. URL : http://journals.openedition.org/narratologie/7687 ; DOI : 10.4000/narratologie. 7687

This text was automatically generated on 15 November 2019.

Article L.111-1 du Code de la propriété intellectuelle. 


\title{
Orient imaginé/Orient vécu chez les Juifs germanophones (yekkes) en Palestine/Israël
}

\author{
Patrick Farges
}

[Israel] blickt nach Westen auf das Zentrum, auf Europa (...). In geographischen Begriffen wird der

Staat Israel als Peripherie Europas definiert.

Yotam Hotam ${ }^{1}$

Rendre compte d'une histoire personnelle suppose que l'on montre aussi comment elle a été accaparée par les puissances de l'imaginaire, porteuses de fables et de vérités qu'ignore (et peut-être doit ignorer) l'histoire collective, celle qui nous sert d'étai symbolique et de lien social.

Claude Burgelin ${ }^{2}$

\section{Introduction}

1 Comme d'autres expériences du monde, l'expérience de la migration et de l'exil est éminemment spatiale, puisqu'elle inclut un voire plusieurs déplacements : déplacement des corps dans l'espace bien sûr, mais aussi déplacement des repères et du cadre socioculturels. L'espace de la migration est un espace construit - notamment par la narration rétrospective - où interagissent les représentations héritées de «là-bas » et les représentations acquises «ici». Si la migration est donc spatiale, les récits de migrants ou plutôt les narrations produites par l'expérience d'une vie post-migratoire gagnent à être (re)lues sous l'angle de la spatialité. Comment un espace d'exil ou de migration peut-il se constituer dans ou par la narration ${ }^{3}$ ?

2 L'espace vécu (erlebter/erfahrener Raum) par les personnes contraintes de fuir l'Allemagne nationale-socialiste a été profondément bouleversé par leur migration - $a$ 
fortiori lorsqu'elles se sont retrouvées dans un pays lointain comme la Palestine mandataire ${ }^{4}$ qu'elles ne connaissaient pour la plupart que très imparfaitement. On estime qu'environ 90000 juifs germanophones émigrèrent en Palestine mandataire dans les années 1930 au cours de ce que l'historiographie sioniste a nommé la "cinquième aliya " $^{5}$. Le présent article est tiré d'une étude plus large sur les Juifs germanophones en Palestine/Israël après 19336. Dans le cas de la Palestine mandataire, la migration juive n'était en rien une migration « ordinaire » - si tant est qu'une migration puisse l'être. D'une part, il s'agissait d'une migration forcée après exclusion de la communauté nationale; d'autre part, contrairement à d'autres destinations d'exil pour les Juifs germanophones, la Palestine avait un statut particulier : aller vers Eretz Israel constituait en effet un retour symbolique (une aliya) vers une patrie d'appartenance, et elle mettait fin symboliquement à la condition d'exil (galout). En réalité pourtant, l'acculturation en Palestine mandataire fut tout aussi malaisée qu'au sein d'autres sociétés d'accueil après 1933. Par-delà le choc de l'arrivée, l'acculturation des Juifs germanophones est aussi passée par leur participation à l'imaginaire de la terre d'accueil, et notamment son imaginaire spatial. La perception du changement étant tout à la fois une opération de reconnaissance partielle et de prise de distance réflexive, les Juifs germanophones en Palestine / Israël ont dû réviser leurs "cartes mentales» héritées du vieux continent et acquises par le biais de diverses instances de socialisation comme les mouvement de jeunesse, les atlas scolaires ${ }^{7}$, ou la littérature de jeunesse et les films.

3 Après quelques remarques concernant l'histoire de la notion de "carte mentale " (mental map) et sa pertinence en dehors du champ des sciences spatiales, il s'agira de comprendre ce qui a permis aux Juifs germanophones d'imaginer l'espace de l'«Orient» et de le mettre à leur portée, tout en mettant en avant les modalités de l'insertion du «moi » dans cet imaginaire, ainsi que les procédés narratifs ayant gouverné cette opération. Mon étude repose en effet sur des sources narratives et largement autobiographiques: récits de vie, entretiens d'histoire orale, correspondances et autres ego-documents. Il s'agira donc aussi d'interroger conjointement la spécificité de ces sources en histoire pour appréhender la construction de l'espace par la narration. Les entretiens d'histoire orale et les egodocuments permettent aux narrateurs de contextualiser l'univers de l'expérience vécue et reformulée, tant sur le plan biographique que sur le plan historique, les deux plans étant fortement imbriqués dans l'opération narrative. Les récits de vie sont donc l'expression d'une expérience individuelle, mais qui incorpore des aspects et des schémas socio-historiques partagés situés dans un espace et un temps précis. Ils donnent donc accès à une réalité historique complexe ${ }^{9}$.

\section{Un imaginaire spatial hérité}

4 La perception du monde par les acteurs de l'Histoire est organisée notamment par les " cartes mentales » (mental maps ${ }^{10}$ ) qu'ils véhiculent, et qui affleurent parfois dans leurs récits de vie. Si la distance géographique est mesurable, la distance psychologique imaginée ou perçue, elle, ne l'est pas: les "cartes mentales", qui rendent une expérience imaginée du monde, sont alors un instrument pour tenter de la mettre en mots $^{11}$. Elles sont l'expression cartographique d'une représentation subjective de l'espace et permettent d'avoir accès - même si cet accès n'est que limité - aux 
références cognitives spatiales de l'individu: structuration subjective de l'espace, jeu entre exo-centrage et ego-centrage, univers métaphorique etc. La traversée d'une ou plusieurs frontières est à la fois un événement réel et un événement imaginé. C'est aussi une opération de comparaison, de reconnaissance partielle et de distanciation.

Même lorsque le départ vers Eretz Israël avait fait l'objet d'une " préparation » sioniste préalable (hakhshara), le pays d'accueil restait fondamentalement étranger et offrait aux esprits aventuriers et pionniers la possibilité imaginée de se mesurer aux forces vitales de la nature. La Palestine pouvait être le lieu de l'échec ou bien au contraire du dépassement de soi, du risque extrême et parfois même de la transgression des codes culturels. L'arrivée en Palestine mandataire était par ailleurs précédée d'une imagination des lieux, d'une pré-science ou d'une pré-conscience plus ou moins complète, voire parfois très floue, d'un «Orient » imaginé... que venaient confirmer les impressions à l'arrivée. Wolf Kaiser a retracé l'imaginaire de l'Orient (et de la Palestine en particulier) tel qu'il existait dans le monde germanophone, en travaillant à partir des récits de voyages d'auteurs juifs entre 1900 et 1939. Ainsi se dessine un récit dominateur et colonial de la différence culturelle, où les Arabes, tout comme peut-être davantage encore les Juifs orientaux, font partie des éléments de décor, tandis que les personnages Juifs européens ont une voix, une identité et une existence narrative attestée ${ }^{12}$. Kaiser conclut que chez les auteurs juifs de langue allemande prédomine une vision orientalisante et stéréotypée qui accentue l'étrangeté d'Eretz Israel. Rares sont les moments où le mode de vie local est valorisé en tant que tel sans faire l'objet d'une comparaison totalisante insistant sur une inégalité fondamentale.

Dans une pure tradition orientalisante, l'imagination ambivalente de la Palestine «levantine» est donc celle d'une construction géopolitique et culturelle, et plus rarement d'un lieu réel et incarné13 ${ }^{13}$ On retrouve cela chez Theodor Herzl lui-même lors de sa visite à Jérusalem en 1898. Dans son journal, à la date du 29 octobre 1898, il relate l'arrivée fastueuse de nuit dans une Jérusalem mythique et imaginée : l'éclat de l'Orient semble encore offrir des possibilités de renouveau, contrairement à l'Occident en déclin. L'attachement à l'Orient permet ainsi de couper le lien avec la condition diasporique de la galout. Et si pour les autres Européens, l'Orient est l'ailleurs absolu, pour les Juifs, il est à la fois « là-bas » et « ici ». Deux jours après son arrivée toutefois, Herzl écrit : « Lorsqu'à l'avenir, je penserai à toi, Jérusalem, ce sera sans grand plaisir. Ici, dans les ruelles malodorantes sont concentrés les vagues résidus de deux millénaires d'inhumanité, d'intolérance et d'impureté $»^{14}$. Les civilisations antiques du Proche-Orient représentent un élément important de l'imaginaire sioniste. Cela est d'autant plus séduisant que ce même Proche-Orient exerçait au même moment une extraordinaire fascination " orientaliste " sur la culture européenne ${ }^{15}$. Cette fascination s'accompagnait parfois de la vision condescendante d'un Orient sale et repoussant, sous la surface duquel se trouvaient les traces admirables des hautes civilisations disparues. L'exhumation des cultures assyro-babyloniennes par les archéologues allemands n'est d'ailleurs pas étrangère à ce phénomène de réception, comme en témoigne l'intérêt des artistes de l'art national juif naissant, dont les chefs de file se trouvent à Berlin ${ }^{16}$.

7 Une autre influence est évidemment constituée par la culture populaire, par exemple la représentation de l'Orient dans le cinéma naissant ${ }^{17}$. Qu'il s'agisse de récits de voyage d'écrivains ou bien de représentations issues de la culture populaire, on retrouve un même rapport ambivalent à l'Orient. D'un côté, l'«autre » oriental arriéré est rejeté avec condescendance, de l'autre se crée un fantasme qui concerne la liberté des 
comportements, des mouvements et des mœurs en Orient. L'Arabe est représenté comme un être vigoureux, instinctif, physique, originel, mais aussi violent, rude, grossier et parfois grotesque dans sa corporalité excessive. Dans l'art sioniste, l'Arabe semble principalement préoccupé par les sens : il se nourrit, se prélasse, il se baigne. «Primitif », il est proche de la nature et des animaux. Le monde oriental ainsi dépeint par les artistes du foyer national juif des années 1920 montre une société oisive, hédoniste et livrée aux plaisirs des sens. Dans le même temps, les tensions réelles s'intensifient avec la poussée migratoire qui vient augmenter la population juive en Palestine (yichouv). Suite aux premières émeutes arabes de 1929, qui se poursuivent dans les années 1930, l'atmosphère change et l'Orient merveilleux cède peu à peu le pas à l'Orient sordide.

8 Il n'est pas étonnant que l'on retrouve cette tension dans les récits de Yekkes arrivés dans les années 1930. Les premières impressions de l'« Orient » raconté correspondent en effet à ce que l'on s'attend à trouver : au moment de l'arrivée se rejoue donc la scène de la rencontre avec l'Orient imaginé et fantasmé. Le jeune Rudi Hiller, né en 1922, est âgé de 14 ans lorsqu'il débarque avec ses parents en Palestine en avril 1936. L'arrivée dans le port de Haïfa est un choc pour l'adolescent et c'est l'impression d'une régression civilisationnelle qui domine en lui. Hiller évoque les odeurs répugnantes, le bruit, l'étrangeté fondamentale de la scène : "Quel balagan (chaos) dans le port ! Et les cris des Arabes, les odeurs étrangères, une vraie pestilence $»^{18}$. Le chaos ambiant est pourtant décrit en employant un terme typique de l'hébreu moderne, "Balagan », qui est comme une marque discursive de l'intégration ultérieure de Rudi Hiller. Prenons un autre exemple: la description de la ville de Jaffa, l'« arabe», la «levantine», par Schlomo Rülf (né en 1896) dans son autobiographie est similaire. Rülf décrit la ville telle qu'il la perçoit au cours d'un premier voyage de reconnaissance effectué au printemps 1933 (soit quelques semaines après l'arrivée d'Hitler au pouvoir en Allemagne), quelques années avant l'émigration définitive en janvier 1935 : «Jaffa, lorsqu'on avait appris à la connaître, s'est révélée sale, bruyante et levantine ; Tel Aviv au contraire était une ville de taille moyenne, normale et propre, de style européen. (...) Tout était bien exactement comme nous l'avions imaginé ! $»^{19}$ Dans cet exemple comme dans beaucoup d'autres, la réalité vient confirmer l'imagination préalable : Jaffa se révèle telle qu'elle avait été imaginée, et Tel Aviv en revanche correspond bien aux standards attendus, "normaux », d'une ville à l'européenne. La ville sainte, Jérusalem, n'échappe pas à cette grille de lecture. Ainsi dans une lettre à ses parents restés en Allemagne datée de novembre 1935, la jeune Lisa, venue en Palestine par le truchement de l'« aliyat hanoar " (Jugendalijah ou "aliya de la jeunesse »), décrit les quartiers arabes de Jérusalem comme un environnement oriental, périlleux et, en outre, fortement sexualisé. Rencontrer l'«Arabe " y est décrit comme la confrontation avec un fauve, tout à la fois fascinant et dangereux. Dans le même temps, la jeune Lisa exprime vis-àvis de ses parents restés en Allemagne son propre courage - sa supériorité aussi -, sa détermination de jeune pionnière, son exaltation du voyage et ses velléités aventurières, autant d'éléments qui correspondent par ailleurs au "grand récit» sioniste qu'on lui inculque dans l'organisation de l'aliya de la jeunesse :

Ma soif d'aventure a été quelque peu freinée. Lors de mes promenades en solo, je me suis retrouvée dans le quartier arabe, à l'intérieur des murs de Jérusalem, toujours plus vides. J'ai entendu siffler derrière moi. J'accélère le pas. Comme la rue est vide, je fais brusquement volte-face. Un jeune Arabe d'environ 18 ans me frôle en faisant des gestes explicites et il me bouscule légèrement. Alors que je reprends mon souffle pendant un instant, il bondit sur moi par derrière comme un félin. - 
Deux coups de poing assénés dans son ventre arabe suffisent certes à la faire déguerpir, mais je dois avouer qu'après cela, et pendant une heure, je ne me suis pas aventurée, ne fût-ce que dans un commerce arabe. ${ }^{20}$

9 L'Orient est donc cette terre de rencontre avec l'étranger certes bien réel, mais dans le même temps fantasmé ; c'est également un espace concret où les pionniers sionistes (haloutsim) font leurs preuves.

10 La rencontre avec l'« Arabe » est l'un des topoi importants des récits de vie, dans la mesure où ce moment biographique revêt une importance d'ordre idéologique et historique : la rencontre entre les pionniers juifs et les «Arabes » est donc l'un des moments de confrontation entre le fantasme et la réalité. Mais le récit de cette rencontre reste fortement marqué par le fantasme. Comme le rappelle Rakefet SelaSheffy, il existe une véritable "folklore» concernant le choc culturel des Yekkes confrontés à la vie en Palestine ${ }^{21}$. En 1935, le jeune Fredi décrit à ses parents en Allemagne un village arabe. Au fil de la lettre, le récit de l'adolescent se focalise de plus en plus et l'espace décrit se concentre sur un groupe d'hommes arabes, puis finalement sur un seul Arabe « enturbanné » et « au regard noir » et « perçant »:

Et finalement, nous sommes arrivés au village. Au village! Voilà à quoi ça ressemble : on voit une maison en pierre, qui en Allemagne serait une ruine, mais qui, ici, est un palais. Tout autour, de nombreuses huttes en terre. C'est affreux, gris, sombre, sale. Après un temps, nous croisons un groupe d'Arabes. (...) J'ai soudain l'idée de m'acheter un poignard arabe. (...) C'est une vision peu agréable que de voir arriver un Arabe, un poignard de trente centimètres à la main, et qui le soupèse et joue avec. Si vous aviez vu cet homme : des yeux noirs, un turban, une longue tunique - une vision sauvage. J'ai réussi à négocier pour faire descendre le prix du poignard de 40 à 20 piastres et je reparti radieux. Arrivé en bas, je me suis rendu compte que ma montre avait disparu. L'Arabe me l'a certainement arrachée avec une pince. Il a sans doute pensé qu'elle avait de la valeur. Si je l'avais pris sur le fait, j'aurais tout de suite mis le poignard à contribution - ou bien je lui aurais asséné un de ces coups. ${ }^{22}$

11 L'espace habité par les Arabes est ici clairement dévalorisé ; la narration est clivée et binaire à la manière d'un conte. Quant à l'Arabe lui-même, habitant cet espace et façonné par lui, il est décrit comme l'ennemi, d'abord superbe et menaçant, mais finalement sournois et cherchant à «rouler» le jeune pionnier. Dans la plus pure idéologie sioniste enfin, le jeune pionnier, exemple du « Nouveau Juif » musclé et prêt à se défendre, n'aurait eu aucun mal à se mesurer physiquement à lui.

\section{Un imaginaire spatial approprié : cultiver le désert}

Non seulement la Palestine est cette terra incognita à la fois étrange et étrangère ; par bien des aspects, notamment par le truchement de l'imaginaire spatial, la Palestine est également décrite dans les récits de Yekkes comme une tierra inculta, c'est-à-dire une terre inhospitalière, qui n'est pas mise en valeur et qui par conséquent n'appartient à personne ${ }^{23}$ : une terre sans agriculture mais aussi sans culture. C'est ce qu'exprime Alexander Cohn de manière radicale en entretien. Alexander Cohn, né en 1912 à Lübeck, s'est rendu une première fois en Palestine en 1931 avant d'émigrer définitivement en 1933 :

[Q]uoi qu'il en soit, je continue de penser que nous sommes meilleurs que les Arabes. Je peux témoigner de ce à quoi ressemblait le pays à notre arrivée. Un désert, mais pas comme le Sahara. Ici, des terres fertiles étaient désertifiées. (...) En 
tout cas, tout ce qui a été construit ici, a été construit par nous. Quand vous regardez les villages arabes aujourd'hui, avec ces grandes maisons qu'ils construisent - tout cela grâce à nous. Si les Arabes étaient restés seuls, ils seraient restés aussi incapables qu'il y a cent ans. Tout est grâce à nous, ça, c'est clair. ${ }^{24}$ Israël, des études critiques ont, depuis les années 1980, remis en question le "grand récit » de l'historiographie, la société et l'identité nationale israéliennes. Cette remise en question a reçu l'étiquette de "post-sionisme ${ }^{25}$ et a notamment été portée par ceux que l'on a appelés les «nouveaux historiens » israéliens : d'abord Simha Flapan, Benny Morris, Tom Segev, Ilan Pappé et Avi Shlaïm, puis ensuite Baruch Kimmerling, Idith Zertal, Shlomo Sand et Uri Ram ${ }^{26}$. En pointant notamment les fronts intersectionnels de domination traversant la société israélienne, qui a marginalisé certains groupes, dont les Arabes et les Juifs orientaux par exemple, cette "nouvelle histoire" a voulu interroger le mythe fondateur sioniste et penser la possibilité d'un pluralisme et d'un multiculturalisme de la nation juive. Étudiant de manière critique les ressorts imaginaires du cinéma israélien, Ella Shohat a ainsi mis en évidence la force du discours orientaliste et colonial dans les films et scénarii sionistes en Palestine mandataire, avant même la création d'Israël. La Palestine y apparaît comme une terre désertique, primitive et improductive attendant d'être "révélée » et fécondée par les pionniers juifs venus d'Europe. La caméra incarne selon Shohat plus généralement l'œil du nouvel arrivant, Yekke par exemple, qui décrit (et donc construit narrativement) l'espace oriental et les Arabes qui le peuplent ${ }^{27}$.

On aboutit ainsi au grand récit pionnier où le halouts, le « Nouveau Juif » pionnier - un homme ou en tout cas une figure virilisée - affronte les marais et les maladies (malaria ou typhus), mais aussi les ennemis arabes, l'arme dans une main, la charrue dans l'autre. En cela, le Nouveau Juif exécute les mêmes gestes mythiques que ses ancêtres bibliques. Cette dimension est particulièrement soulignée dans le récit autobiographique de Michael Evenari, né sous le nom de Walter Schwarz en 1904 à Metz. Exclu de l'Institut de Botanique de l'Université technique de Darmstadt parce qu'il est juif, il émigre dès avril 1933. Actif dans les mouvements sionistes allemands, son émigration est placée sous le signe de l'adhésion au sionisme. Il devient enseignant à l'Université Hébraïque de Jérusalem et se spécialise dans les nouvelles méthodes d'irrigation du désert du Néguev. Il deviendra même vice-président de l'Université Hébraïque. Son autobiographie s'intitule Que le désert porte des fruits (Und die Wüste trage Frucht) : le titre, sous forme injonctive, est une réminiscence biblique. Il s'agit d'un récit à la gloire du projet sioniste, qui procède à un important lissage narratif des difficultés rencontrées :

Au moment de mon immigration en Palestine en 1933, le pays m'était étranger. Et pourtant, je me sentais comme à la maison. Ici, entre Juifs travaillant dans le bâtiment ou l'industrie, policiers, constructeurs de routes ou agriculteurs, il n'y avait pas d'antisémitisme. (...) J'étais un individu parmi des égaux, sûr de moi, fier d'être juif. Nous étions des idéalistes - un mot décrié aujourd'hui -, animés par la flamme de construire notre propre Etat à venir. J'étais heureux. Mais la vie quotidienne n'était pas simple. Le pays avait été délaissé depuis des centaines d'années, la forêt d'origine avait été détruite, les paysages s'étaient désertifiés. De grandes parties du pays étaient des marécages infestés par la malaria. ${ }^{28}$

Comme dans d'autres récits de type colonial, l'imaginaire spatial développé est celui d'une terre vierge qui attend d'être rendue fertile par le pionnier, le sol, vierge, est là pour être fécondé. 
Cela conduit même parfois à un processus genré de sexualisation de l'espace. Dans certains récits de Yekkes, l'imaginaire pionnier dominant la terre orientale inclut jusqu'à la sémantique de l'extase sexuelle. La sexualisation du rapport colonial à la terre orientale se trouve ainsi au cœur d'un récit autobiographique de Fritz Joseph Heidecker, né en 1912, émigré en 1934 et très actif dans le collectivisme sioniste. Le récit, qui se veut édifiant, relate les "années pionnières " suivant l'arrivée en Eretz Israël. Dans l'extrait choisi ci-dessous, il est question du forage d'un puits, action hautement stratégique pour "faire fleurir" le désert. L'extrait met en scène un collectif d'hommes, des haloutsim, qui peinent à pénétrer la terre étrangère avec l'outil de forage, de forme oblongue, objet éminemment sexualisé et rendu anthropomorphique. Les couches de terre cèdent bruyamment les unes après les autres, apportant aux pionniers un sentiment de délivrance, de puissance et de jouissance. Chaque mètre gagné est vécu comme une victoire personnelle de l'homme conquérant sur la terre orientale (féminisée), d'abord réticente :

Le puits était maintenant consolidé de pierres sur une profondeur de vingt mètre. Là, la seconde partie du travail consistait à faire rentrer des tubes de fer longs de quatorze pouces dans la terre, toujours plus profondément (...).

Les épaules couvertes de sueur, quatre travailleurs marchaient en rond, deux de chaque côté, actionnant ainsi les leviers d'acier et enfonçant la foreuse dans le sable qui cédait dans un couinement fracassant. Puis le cylindre d'acier haut comme un homme se relevait dans un souffle victorieux et vomissait ce qu'il avait dans le ventre, puis il balançait au bout du câble qui se tendait derechef, jusqu'à ce que la pointe se retrouve au-dessus de l'embouchure du puits. Et il plongeait à nouveau. Nous marchions en rond encore et toujours, les épaules endolories. De temps en temps, il nous fallait enfoncer davantage le tube d'acier dans les couches vaincues. (...) Jamais auparavant je n'avais ressenti aussi clairement la jouissance de voir ainsi de mes yeux le travail progresser. Chaque mètre gagné était comme un triomphe personnel ${ }^{29}$.

17 Cette scène a le statut de moment fondateur. Elle montre l'appropriation et la domination de l'espace colonisé par un collectif d'hommes, les pionniers juifs. Ces derniers, galvanisés par la tâche mythique qu'ils réalisent, en ressentent individuellement une satisfaction et un plaisir qui proviennent aussi de la sexualisation collective - réprimée - de leurs actes. On est ici placé au cœur du problème de l'énonciation historique en contexte colonial, une question déjà soulevée par Michel de Certeau: dans la deuxième édition de L'Écriture de l'histoire, il commente en effet une représentation du conquérant Amerigo Vespucci, l'homme blanc et armé, " découvrant » (c'est-à-dire révélant tout en dénudant) l'Amérique ${ }^{30}$. Dans ce texte, De Certeau entend insister sur les rapports de pouvoir mobilisés dans l'écriture de l'Histoire depuis la découverte coloniale du Nouveau Monde. L'Histoire peut dès lors être vue comme un rapport de domination sexualisée que le sujet de l'énonciation, l'explorateur européen, exerce sur l'objet du texte qu'il rédige : le corps nu et féminisé de l'« autre » colonisés ${ }^{31}$. La nudité y est un enjeu important : tant l'espace du nomade colonisé, «lisse » (selon la terminologie géo-philosophique de Gilles Deleuze), que le corps de l'autre, nu, sont exempts de marques de civilisation ${ }^{32}$.

\section{Un espace « troué »?}

Dans l'extrait précédent, le forage du puits équivaut à une forme de prise de possession coloniale. L'accès au sous-sol, qu'il s'agisse de l'eau ou bien des ressources naturelles ${ }^{33}$, 
requiert de trouer et de baliser l'espace «lisse» et jusque-là insaisissable de l'« Orient », réticent. Selon Deleuze et Guattari, l'espace colonisé (par l'Occident ou la pensée occidentale) devient ainsi un espace "strié ", c'est-à-dire nommé, mesuré, codifié, compartimentés ${ }^{34}$. L'historiographie sur les Yekkes, qui connaît un véritable engouement depuis le milieu des années 1990, a beaucoup insisté sur ce que les nouveaux arrivants venus d'Allemagne et d'Europe centrale avaient modifié dans l'espace du yichouv (la communauté juive en Palestine mandataire), et, plus généralement, dans la société israélienne. L'historien israélien Yoav Gelber, auteur au début des années 1990 de la première grande étude sur la " cinquième aliya ", appelée aussi « deutsch(sprachig)e Alija» (ou aliya ha-Germanit), fait des Yekkes les vecteurs de la modernisation d'une Palestine qui jusqu'à leur arrivée, serait restée arriérée, nonoccidentalisée et «spartiate ». Il met en avant les qualités intrinsèques de ce groupe, qui les distinguerait d'une part des autres Juifs du yichouv (notamment les Juifs originaires d'Europe orientale), d'autre part des « Orientaux» :

La diversité socio-économique et professionnelle des immigrants allemands, leur sens de la famille, leur pensée sociale et leurs habitudes de consommation ont modifié drastiquement le mode de vie juif en Palestine. En l'espace de quelques années, ils ont transformé une communauté de pionniers, fondée sur une idéologie spartiate et élitiste, en une société pluraliste et cultivée. C'était là le premier pas vers la société du bien-être et l'Etat-Providence qu'Israël allait être ${ }^{35}$.

Or les Yekkes sont arrivés à un moment - les années 1930 - où l'espace de la Palestine mandataire avait déjà largement été balisé (par les structures ottomanes anciennes et l'administration britannique notamment) et où le yichouv était lui aussi largement organisé et constitué. Les Yekkes sont arrivés dans une Palestine mandataire qui s'était organisée sans eux, même si l'historiographie yekke a beaucoup insisté sur le rôle de «bâtisseurs» des Juifs germanophones des années 1930. C'est ce qu'exprime Friedrich Reichenstein, né en 1906 à Herne et immigré en Palestine en $1935^{36}$, dans son récit autobiographique : « La contribution des immigrants venus d'Europe centrale a modifié le caractère du yichouv et a fait d'Israël un îlot de culture et de civilisation au milieu d'un Orient qui, par comparaison, avait du retard. ${ }^{37}$ Ce propos n'est pas isolé.

Dans l'historiographie et la mémoire yekke, se déploie en réalité la vision d'un espace israélien «troué », fait de pleins, en particulier les lieux de mémoire yekke considérés comme " civilisés ", et de taches "blanches ", qui restent autant de tierras incultas. Ainsi existe-t-il une mémoire et une historiographie spécifiques concernant différentes Sprachinseln $^{38}$, selon l'expression d'Anne Betten, ces îlots de culture (qui sont aussi des îlots de refuge de la langue allemande). C'est le cas du quartier de Rehavia à Jérusalem, avec ses "grands hommes" incarnant le Bildungsbürgertum juif (Hugo Bergmann, Gershom Scholem, Martin Buber ou encore Werner $\mathrm{Kraft}^{39}$ ), ou bien encore de la ville de Nahariya au nord d'Israël, fondée en 1934 par des Juifs allemands ayant racheté des terres à des grands propriétaires fonciers arabes ${ }^{40}$. C'est aussi le cas du Mont Carmel à Haïfa ${ }^{41}$ ou du "Kanton Ivrit ", c'est-à-dire le nord de Tel Aviv autour de la rue Ben Yéhouda $^{42}$. Ces lieux «pleins", auxquels il faut sans doute rajouter le kibboutz Dalia ou encore le moshav Sde Warburg, ont conduit à produire une mémoire de la présence yekke en Palestine/Israël. C'est donc une lutte symbolique autour d'une distinction socioculturelle spatialisée qui se joue. Au sein de cet espace "troué », se pose alors le problème des "autres ", c'est-à-dire de ceux qui occupent le reste du territoire. Qui sont les «autres» de la mémoire et l'historiographie yekke? Les Arabes? Les Juifs d'Europe orientale? Les Juifs orientaux (mizrahim)? 
21 L'une des questions cruciales est bien évidemment constituée par les relations entre les Yekkes et les populations arabes. Les années 1930 sont une période de tensions grandissantes entre Juifs et Arabes. Avec l'afflux de Juifs d'Europe au cours de la « cinquième aliya », les populations arabes se révoltent, parfois violemment, comme entre 1936 puis 1939, afin de faire réagir l'administration mandataire britannique. Les Yekkes ont vécu cela au moment de leur arrivée. Ils furent aussi les témoins de la Guerre de 1948 (appelée "Guerre d'indépendance » dans l'historiographie israélienne). La création de l'État d'Israël s'est assortie d'une complète redéfinition territoriale et de migrations de populations, un événement connu dans la mémoire palestinienne arabe sous le nom de «nakba» (catastrophe). Dans le récit de vie qu'il donne en 1991, Ernst Pfeffermann, né en 1917 à Berlin, développe une pensée spatiale et territoriale de la relation entre Juifs et Arabes. Il reprend notamment à son compte le récit nationaliste israélien selon lequel les Arabes auraient, en quittant leurs lieux d'habitation en 1948 (c'est-à-dire par leur « absence $»^{43}$ ), abandonné leurs terres et par conséquent tout droit à la propriété :

Les Arabes (...), ils ont déguerpi, car entre temps il y a eu la création du State of Israel, n'est-ce pas? Les Arabes ont tous déguerpi et ils ne sont jamais revenus. (...) Si j'évoque les Druses, c'est parce que les Druses n'ont pas déguerpi. Ils n'étaient pas concernés et du point de vue de la sécurité, ils ont toujours été considérés comme les égaux des Juifs. (...) Je ne hais pas les Arabes, au contraire : je pense qu'il nous faut cohabiter. (...) Et nous sommes comme une excroissance, une plaie au MoyenOrient. Les Arabes ne comprennent que deux langues, l'arabe et la langue du plus fort. Il est impossible de s'adresser à eux dans une autre langue. (...) Nous n'avons pas la mentalité d'ici. Nous ne sommes pas des Orientaux. Je ne parle pas des quelques Juifs qui, avant, vivaient en Afrique du nord ou bien quelque part avec des Arabes. La majorité vient d'autres pays, et nous ne sommes pas prêts à adopter le mode de vie arabe. Et par conséquent, notre culture et nos idéaux ne sont pas adaptés au Moyen-Orient. ${ }^{44}$

Dans ce récit, la problématique de l'espace «troué » est également présente à mon sens. Les terres «abandonnées » par les populations arabes sont devenues autant de lieux d'un possible investissement civilisationnel clairement identifié comme non-oriental (« Nous n'avons pas la mentalité d'ici. Nous ne sommes pas des Orientaux.»). La conséquence en est qu'Ernst Pfeffermann voit la présence juive(-allemande) comme une excroissance (il dit en allemand : « un furoncle ») au Proche-Orient. Curieusement, dans cet extrait, le rapport habituel à l'Orient sale voire malade (et donc dangereux) est renversé, dans la mesure où ce sont les Juifs eux-mêmes qui sont associés à la maladie (au furoncle). Au cours de l'entretien, et en réaction à l'interrogation de l'intervieweuse, Ernst Pfeffermann revient d'ailleurs sur cette métaphore, la jugeant trop « extrême »: " Peut-être ai-je employé une expression trop extrême en parlant de furoncle au Moyen-Orient. $»^{45}$

Aux yeux des Yekkes, les "autres ", ce sont aussi les Juifs d'Europe orientale bien sûr (les Ostjuden), mais surtout les Juifs orientaux, ceux qui étaient présents de longue date et ceux qui affluent en Israël après 1948 en provenance du Yémen ou d'Irak. Ces derniers sont vus comme représentant une menace pour l'homogénéité de la nation juive $^{46}$. Les stigmates de l'« orientalité » leur collent à la peau : ils parlent arabe, ne s'identifient pas à la culture occidentale européenne et l'on remarque leur couleur de peau plus foncée ${ }^{47}$. En cela, le travail de distinction spatiale entre Juifs orientaux et Yekkes - qui participent de l'hégémonie ashkénaze marquant la construction de la nation israélienne - rejoint un vieux débat à l'intérieur du mouvement sioniste afin de 
montrer que les Ashkénazes ne sont pas des «Sémites ». En cela, les Yekkes, bien qu'ils ne se soient pas pliés à certaines des injonctions du sionisme, en ont adopté l'imaginaire spatial, largement orientaliste. L'universitaire israélien Amnon RazKrakotzkin rappelle d'ailleurs à quel point les différentes lignes de domination socioculturelles en Israël s'expliquent par des schémas orientalistes :

[Le sionisme] peut se lire comme l'expression extrême d'un désir d'assimilation juif dans le grand récit occidental, celui des Lumière et de la rédemption. (...) Les sionistes ont développé tout un éventail d'attitudes envers l'Orient et les Arabes, qui va du désir romantique au déni le plus total. Mais tous sont restés dans le cadre de la dichotomie orientaliste et tous ont concouru à créer le Juif nouveau, que le sionisme a voulu définir comme un Européen, non un Oriental. Dans ce processus, les mêmes termes qui avaient été employés contre les Juifs en Europe (...) ont été utilisés afin de recréer une collectivité juive nationale et ils ont servi - dans des modalités différentes toutefois - à qualifier les Palestiniens ainsi que les Juifs venant de pays arabes. L'orientalisme est intrinsèquement lié à toutes les dichotomies utilisées communément pour décrire la société israélienne: Juifs/ Arabes, Ashkénazes/Mizrahim, de même que laïque/religieux. ${ }^{48}$

\section{Conclusion}

Pour les Yekkes, le processus d'acculturation est aussi passé par une participation critique à l'imaginaire israélien en train de se construire, ainsi que par l'adaptation de leurs schèmes interprétatifs aux représentations disponibles et socio-culturellement acceptées, issues du sionisme. Il s'agissait ici de comprendre ce qui leur a permis d' imaginer leur nouvelle terre d'accueil et de la mettre à leur portée spatialement. Dans le même temps, il s'agissait de mettre en avant les modalités de l'insertion de leur " moi » dans l'espace post-migratoire, ainsi que les procédés narratifs gouvernant cette opération. Cette insertion, cette appropriation du pays d'accueil par l'imaginaire Deleuze dirait: cette «reterritorialisation»- ont pris la forme d'une formulation narrative du récit de vie, toujours déjà auto-créatrice.

\section{NOTES}

1. « [Israël] regarde vers l'ouest sur un centre qu'est l'Europe (...). En termes géographiques, l'État d'Israël se situe dans la périphérie de l'Europe. » Yotam Hotam, « Im Perimeter Europas : Über die "deutsch-jüdische" israelische Gesellschaft und eine Gegenstrategie », dans Moshe Zimmermann \& Yotam Hotam (éds.), Zweimal Heimat. Die Jeckes zwischen Mitteleuropa und Nahost, Francfurt/M., 2005, p. 109.

2. Claude Burgelin, «Écriture de soi, écriture de l'Histoire : esquisses autour d'un conflit », in Jean-François Chiantaretto (éd.), Écriture de soi, écriture de l'histoire, Paris, 1997, p. 99.

3. Cette question rejoint évidemment les préoccupations ayant trait à un spatial turn au sein des études sur l'exil (Exilforschung) et les migrations. Sur les «tournants » en sciences humaines et sociales, voir Doris Bachmann-Medick, Cultural Turns. Neuorientierungen in den Kulturwissenschaften, Reinbek bei Hamburg, 2006, p. 284-328. Sur le «tournant topographique », 
voir plus spécifiquement Stefanie Stockhorst, «"Raum” als kulturwissenschaftliches Paradigma. Begriffliche, methodische und thematische Perspektiven für eine Germanistik im Zeichen des "topographical turn" ", dans Françoise Lartillot \& Ulrich Pfeil (éd.), Constructions de l'espace dans les cultures d'expression allemande, Berne, 2013, p. 7-32. Dans le même volume, voir Patrick Farges, «Constructions de l'espace dans les récits de vie d'exilés germanophones au Canada », p. 159-175. 4. La définition de la "Palestine " n'est pas aisée. Ici, le terme désignera le territoire situé à l'Ouest du Jourdain et sous mandat britannique de 1922 à 1948.

5. Vgl. Heid, Ludger : «Palästina/Israel », dans Claus-Dieter Krohn, Patrik von zur Mühlen, Gerhard Paul \& Lutz Winckler (éd.), Handbuch der deutschsprachigen Emigration 1933-1945, Darmstadt, 1998, p. 349-358.

6. Voir Patrick Farges, «Jeckes in Palästina/Israel. Versuch einer Männlichkeitsgeschichte », dans Katja Schubert \& Laurence Guillon (éd.), Deutschland und Israel/Palästina von 1945 bis heute, Würzburg, 2014, p. 53-73 ; Patrick Farges, « Récits de vie, stratégies narratives et construction de la masculinité. Le cas des exilés germanophones en Palestine/Israël », dans Bernard Banoun, Anne Tomiche \& Mónica Zapata (éd.), Fictions du masculin dans les littératures occidentales, Paris, 2014, p. 83-96.

7. Longtemps négligée, la dimension historique des cartes produites dans les atlas scolaires ou les cours de géographie doit en effet aujourd'hui être interrogée. Voir Christof Dipper \& Ute Schneider, Der Raum und seine Repräsentation in der Neuzeit, Darmstadt, 2006.

8. Notamment les quelque 150 entretiens de l'«Israel-Corpus $-1^{\mathrm{e}}$ génération » (IC1) constitué dans les années 1990 par une équipe de recherche germano-israélienne dirigée par Anne Betten (Université catholique d'Eichstätt puis Université de Salzbourg) et Miryam Du-nour (Université Bar-Ilan, Israël). Voir la présentation du corpus sur le site de l'Institut für Deutsche Sprache de Mannheim (section Gesprochenes Deutsch) (http://agd.ids-mannheim.de/IS--_extern.shtml). Voir aussi Anne Betten \& Miryam Du-nour (éd.), Sprachbewahrung nach der Emigration - Das Deutsch der 20er Jahre in Israel, t. I \& II (Phonai 42 \& 45), Tübingen 1995 \& 2000.

9. Sur les enjeux de l'histoire orale, voir Alessandro Portelli, « The Peculiarities of Oral History », in History Workshop Journal, $\mathrm{n}^{\circ} 12.1$ (1981), p. 96-107; Danièle Voldman, «L'histoire orale entre science et conscience ", in Vingtième siècle. Revue d'histoire, n² 25 (1990), p. 113-115; Donald A. Ritchie, Doing Oral History, Oxford, 2014 ; Fabrice D’Almeida \& Denis Maréchal (dir.), L'histoire orale en questions, Paris, 2014.

10. Arrêterons-nous brièvement sur le concept de mental map. Au cours des années 1970, la géographie "classique » a dû faire face à une impasse théorique (absence de nouveaux concepts) et pratique (absence de nouveaux terrains). De nouvelles directions sont alors apparues, comme la géographie des représentations, la "géographie de la perception" d'inspiration anglosaxonne, ou encore la pensée d'Armand Frémont sur les " espaces vécus " (La région, espace vécu [1976], Paris, 1999). Selon cette "nouvelle géographie», l'espace représenté reflète les caractéristiques mentales et cognitives de l'esprit humain et, de surcroît, se charge de valeurs et d'émotions, portant ainsi la marque des codes culturels et idéologies en vigueur, ainsi que de ce que l'on pourrait appeler la « conjoncture affectuelle» des individus. Sur le concept de «mental map », voir l'article « Carte mentale » dans Jacques Lévy et Michel Lussault (éd.), Dictionnaire de la géographie et de l'espace desm sociétés, Paris, 2003, p. 132 sq. Voir aussi Guy Di Méo, Géographie sociale et territoires, Paris, 1998, en particulier p. 27-35.

11. Abraham André Moles et Elisabeth Rohmer, Psychologie de l'espace, Paris, 1972.

12. Wolf Kaiser, Palästina - Erez Israel. Deutschsprachige Reisebeschreibungen jüdischer Autoren von der Jahrhundertwende bis zum Zweiten Weltkrieg, Hildesheim-Zurich, 1992, p. 488.

13. Voir Dominique Trimbur (éd.), Europäer in der Levante - Zwischen Politik, Wissenschaft und Religion (19.-20. Jahrhundert), Munich, 2004 ; Olivier Baisez, Architectes de Sion : la conception par les sionistes allemands de la colonisation juive en Palestine (1896-1919), Paris, 2015. 
14. «Wenn ich künftig deiner gedenke, Jerusalem, wird es nicht mit Vergnügen sein. Die dumpfen Niederschläge zweier Jahrtausende voll Unmenschlichkeit, Unduldsamkeit und Unreinlichkeit sitzen in den übel riechenden Gassen ». Entrée du 31 octobre 1898, in Theodor Herzl, Gesammelte zionistische Werke, Berlin ${ }^{3} 1934$, vol. III, p. 212. Sur la question controversée de la relation entre Herzl et les Arabes de Palestine, voir Derek J. Penslar, «Herzl and the Palestinian Arabs : Myth and CounterMyth », in The Journal of Israeli History, $n^{\circ} 24.1,2005$, p. 65-77.

15. Voir Edward Said, L'Orientalisme. L'Orient créé par l'Occident [1978], Paris, 2005.

16. Voir Yigal Zalmona, "Vers les confins de l'Orient. L'art sioniste naissant en quête d'une identité hébraïque », dans Laurence Sigal-Klagsbald (éd.), Les Juifs dans l'Orientalisme, Paris, 2012, p. 33-43.

17. Voir Ella Shohat, Israeli Cinema : East/West and the Politics of Representation [1989], New YorkLondres, 2010

18. «Was für ein Balagan (Chaos) am Hafen! Und die lauten Araber, die fremden Gerüche, gestunken hat es. » Cité dans Thorsten Schmitz, «Vierzig Jahre deutsch-israelische Beziehungen : Die Jeckes als Seismografen », dans Süddeutsche Zeitung, 12/04/2005.

19. «Bei näherer Bekanntschaft stellte sich Jaffa als dreckig, laut und levantinisch heraus, dagegen Tel Aviv als eine normale, saubere Stadt mittlerer Größe nach europäischem Muster. (...) Ja, es war alles so, wie wir es uns vorgestellt hatten!» Schlomo Rülf, Ströme im dürren Land. Erinnerungen, Stuttgart, 1964, p. 80.

20. « Meine Abenteuerlust ist etwas gemildert. Ich geriet auf meinen Solopromenaden ins arabische Viertel - zwischen die Stadtmauern Jerusalems, die immer leerer werden. Ich hörte hinter mir pfeifen, gehe schnell weiter. Da kein Mensch mehr zu sehen ist, kehre ich rasch um. Ein etwa 18jähriger Araber schleicht mit bedeutsamen Zeichen an mir vorbei und gibt mir einen leichten Schlag. Ich will gerade aufatmen, da springt er wie eine Katze von rückwärts auf mich. - Zwei gehörige Boxhiebe in seinem arabischen Bauch genügen zwar, um ihn zu verscheuchen. Aber ich muss gestehen, dass ich mich daraufhin für eine Stunde sogar kaum mehr in ein arabisches Geschäft wagte. » Cité dans Rudolf Melitz (éd.), Jeruschalajim, den... Briefe junger Menschen schildern Erez Israel, Berlin, 1936, p. 52.

21. Rakefet Sela-Sheffy, " "Europeans in the Levant" Revisited - German Jewish Immigrants in 1930s Palestine and the Question of Culture Retention», in Tel Aviver Jahrbuch für deutsche Geschichte, $n^{\circ} 41,2013$, p. 43.

22. " Endlich kamen wir ins Dorf. Ins Dorf! Das sieht folgendermaßen aus : Man sieht ein Steinhaus, das in Deutschland als Ruine angesehen würde, hier aber als Palast gilt. Ringsherum stehen lauter Lehmhütten. Scheußlich, grau, dunkel, dreckig. Nach einer Weile trafen wir auf einen Kreis Araber. (...) Plötzlich kam ich auf die Idee, mir einen Araberdolch zu kaufen. (...) Es war ein ungemütlicher Anblick, wie ein Araber mit einem dreißig Zentimeter langen Dolch ankam und ihn spielerisch in der Hand wog. Ihr hättet den Mann sehen müssen : schwarze Augen, Turban, lang Gewänder - ein wilder Anblick. Den Dolch handelte ich von vierzig auf zwanzig Piaster herunter und zog freudestrahlend ab. Unten merkte ich, dass mir meine Uhrkette fehlte. Der Araber muss sie mit einer Zange abgezwickt haben. Er hielt sie sicher für wertvoll. Wenn ich ihn dabei erwischt hätte, würde er sicher den Dolch gleich gebraucht haben - oder ich hätte ihm ein Ding versetzt. » Cité dans Rudolf Melitz (éd.), Jeruschalajim, den... Briefe junger Menschen schildern Erez Israel, Berlin, 1936, p. 142-143.

23. Le terme est évidemment issu de l'imaginaire colonial. Il est en particulier emprunté à la colonisation espagnole de l'Amérique du sud et en particulier au régime des Jésuites. Voir Nicholas P. Cushner, Farm and Factory. The Jesuits and the Development of Agrarian Capitalism in Colonial Quito. 1600-1767, Albany (NY), 1982. On sait par ailleurs l'importance de l'argument de la "terre qui n'appartient à personne " dans les relations entre Juifs et Arabes au moment de la Guerre de 1948 qui a conduit à la naissance de l'État d'Israël. Nous y reviendrons.

24. "[J]edenfalls, ich glaube immer noch, dass wir besser sind als die Araber. Ich kann es bezeugen, wie dieses Land ausgesehen hat, als wir hergekommen sind. Eine Wüste, nicht nur die Wüste Sahara. Hier war fruchtbares Land Wüste. (...) Jedenfalls, wenn irgendetwas gebaut worden ist, haben wir das gebaut. Wenn 
Sie die arabischen Dörfer heute sehen, alle mit den großen Häusern, die sie bauen, nur dank uns; wenn die Araber allein gewesen wären, wären sie so läppisch gewesen, wie sie vor hundert Jahren waren. Alles dank uns, das ist klar. » Entretien avec Alexander Cohn, Ramat Gan, 19/02/2000, dans Hermann Zabel (éd.), In der Erinnerung liegt das Geheimnis der Erlösung. Gespräche mit Israelis deutscher Muttersprache, Essen, 2002, p. 72-73.

25. Voir Laurence J. Silberstein, The Postzionism Debates : Knowledge and Power in Israeli Culture, New York, 1999 ; Laurence J. Silberstein (éd.), Postzionism : A Reader, New Brunswick (NJ), 2008.

26. Voir Ilan Greilsammer, La nouvelle histoire d'Israël, Paris, 1998. Pour une lecture critique de ces «nouveaux historiens », voir Sébastien Boussois, Israël confronté à son passé : Essai sur l'influence de la « Nouvelle Histoire», Paris, 2007 ; Elhanan Yakira, Post-sionisme, post-Shoah. Trois essais sur une négation, une délégitimation et une diabolisation d'Israël, Paris, 2010.

27. Voir Ella Shohat, Israeli Cinema: East/West and the Politics of Representation [1989], New YorkLondres, 2010.

28. «Als ich 1933 in Palästina einwanderte, war das Land anfangs für mich eine fremde Welt. Trotzdem fühlte ich, dass ich nach Hause gekommen war. Hier unter Juden, die Bau- und Fabrikarbeiter und Polizisten waren, Straßen bauten und als Bauern das Land bearbeiteten, gab es keinen Antisemitismus (...). Ich war Gleicher unter Gleichen, selbstbewusst, stolz, ein Jude zu sein. Wir waren Idealisten - heute beinahe ein verpöntes Wort-, voll des Feuers, unser eigenes Land, unseren künftigen Staat aufzubauen. Ich war glücklich. Doch das tägliche Leben war nicht leicht. Das Land war für Hunderte von Jahren vernachlässigt worden, der ursprüngliche Wald vernichtet, die Landschaft öde. Große Teile des Landes waren MalariaSümpfe. » Michael Evenari, Und die Wüste trage Frucht. Ein Lebensbericht, Gerlingen, Bleicher, 1990, p. 156-157.

29. «Der Schacht des Brunnens war jetzt bis in eine Tiefe von zwanzig Metern ausgemauert. Der zweite Teil der Arbeit bestand darin, von seinem Grund aus vierzehnzöllige Eisenrohre in die Erde zu treiben, tiefer und tiefer (...). Mit schweißnassen Schultern drückten vier Arbeiter, je zwei und zwei, im Rundgang um den Schacht mit stählernen Hebeln den Bohrer in den Sand, der sich mit knirschender Wut ergab. Dann fuhr der mannshohe Stahlzylinder empor, während die Winde jubelnd kreischten, und erbrach seinen Fraß, tänzelte am sich wieder straffenden Seil, bis die Spitze über der Schachtmündung stand und tauchte von neuem hinab. Rundum, rundum gingen wir, mit schmerzenden Schultern. Von Zeit zu Zeit mussten wir den Eisenschacht weiter hinabstoßen in die eroberte Schicht. (...) Niemals vorher hatte ich mit solcher Klarheit die Befriedigung empfunden, den Fortschritt einer Arbeit so sichtbar vor Augen zu haben. Jeder neu errungene Meter war wie ein persönlicher Triumph.» Fritz Joseph Heidecker, Die Brunnenbauer. Jüdische Pionierarbeit in Palästina 1934-1939, éd. Erhard Roy Wiehn, Constance, 1998, p. 62. Le manuscrit, rédigé en 1939-1940 en allemand, fut présenté en 1944 à Max Brod, installé à Tel Aviv, qui le trouva «bien écrit et bien senti ». Il ne fut pour autant pas publié à l'époque.

30. Il s'agit d'une gravure de Theodor Galle en 1580 d'après un dessin de Jan van der Straet.

31. Voir Michel de Certeau, L'Écriture de l'histoire, Paris, 1980. L'image était présente dès la première édition de l'ouvrage en 1975 , mais sans le commentaire.

32. Voir Joachim Schlör, "Der nackte und der bekleidete Körper », in Sander L. Gilman, Robert Jütte \& Gabriele Kohlbauer-Fritz (éd.), "Der schejne Jid”. Das Bild des "jüdischen Körpers" in Mythos und Ritual, Vienne, 1998, p. 122-132.

33. C'est d'ailleurs jusqu'à aujourd'hui l'un des aspects cruciaux du problème israélo-palestinien. 34. Gilles Deleuze et Félix Guattari, Mille Plateaux (Capitalisme et schizophrénie, vol. 2), Paris 1980, p. $592 s q$.

35. «Die sozioökonomische und berufliche Vielfalt der deutschen Immigration, ihr Familiensinn, ihre sozialen Ideen und ihre Konsumgewohnheiten veränderten den Lebensstil des jüdischen Palästina drastisch. Innerhalb weniger Jahre verwandelten sie die auf eine spartanisch-elitäre Ideologie gegründete Gemeinschaft von Pionieren in eine pluralistische und kultiviertere Gesellschaft. Das war der erste Schritt $z u$ einer Wohlstandsgesellschaft und zum modernen Wohlfahrtsstaat, zu dem sich Israel später entwickelte.» Yoav Gelber, «Die historische Rolle der mitteleuropäischen Immigration nach 
Israel ", in Magdalene Krumpholz (éd.), Die "Jeckes" in Israel. Der Beitrag der deutschsprachigen Einwanderer zum Aufbau Israels, Bonn 1995, p. 87-88.

36. Un an plus tard, il fonde le journal de langue allemande Yedioth Hayom qu'il dirige jusqu'à ce que la publication cesse en 1964.

37. «Der Beitrag der Einwanderer aus Mitteleuropa verändert den Charakter des Jischuv und macht Israel $z u$ einer Insel von Kultur und Zivilisation inmitten des noch vergleichsweise rückständigen Ostens. » Friedrich Reichenstein, Yedioth Hayom. Ich und meine Zeitung, eine erlebte Geschichte, Tel Aviv, 2007, p. 12.

38. Voir Anne Betten, «Die Sprachinsel der Jeckes », dans Gisela Dachs (éd.), Sprachen. Jüdischer Almanach des Leo Baeck Instituts, Francfort/M., 2007, p. 33-42.

39. Sur le récit archétypal concernant le quartier de Rehavia à Jérusalem, "centre d'une extraordinaire sociabilité juive allemande», voir Dominique Bourel, "Le village "yekke": capitale du judaïsme allemand », in L'Histoire vol. 7, n 378, 2012 (dossier spécial « Jérusalem »), p. 78. Voir aussi David Kroyanker, «Rechavia - Das "Jeckenland" von Jerusalem », dans Moshe Zimmermann \& Yotam Hotam (éd.), Zweimal Heimat. Die Jeckes zwischen Mitteleuropa und Nahost, Francfort/M., 2005, p. 260-266 ; Sonia Goldblum, « Von Jerusalem nach Weimar. Rückblick auf die deutsch-jüdischen Beziehungen in der Zwischenkriegszeit", in Katja Schubert \& Laurence Guillon (éds.), Deutschland und Israel/Palästina von 1945 bis heute, Würzburg, 2014, p. 37-52.

40. Voir le documentaire de David Witzthum, Nahariyade, 2002, 52 Min. Voir aussi Erich M. Lehmann, Nahariya. Ein Beitrag der mitteleuropäischen Einwanderung zum Aufbau des Landes Israel, Nahariya, 1960 ; Klaus Kreppel, Israels fleißige Jeckes. Zwölf Unternehmerportraits deutschsprachiger Juden aus Nahariya, préface de Paul Spiegel, Bielefeld, 2002 ; Klaus Kreppel, Nahariyya - das Dorf der “Jeckes". Die Gründung der Mittelstandssiedlung für deutsche Einwanderer in Eretz Israel 1934/35, Tefen, 2005.

41. Voir Anja Siegemund (éd.), Deutsche und zentraleuropäische Juden in Palästina am Beispiel Haifa in der ersten Hälfte des 20. Jahrhunderts, Berlin, 2016.

42. Selon la légende, on n'y parlait « kaan Ton Ivrit », ce qui en dialecte viennois signifie " pas un mot d'hébreu » et qui se prononce comme «canton Ivrit». Sur Tel Aviv, voir Joachim Schlör, «"... das Großstadtleben nicht entbehren.” Berlin-Tel Aviv : Großstadtpioniere auf der Suche nach Heimat », in Exilforschung. Ein internationales Jahrbuch, nº 13 ("Kulturtransfer im Exil”), 1995, p. 166-183.

43. En vertu d'une loi israélienne de 1950, les Palestiniens arabes ayant abandonné leur propriété entre le 29 novembre 1947 (date du vote par l'ONU du plan de partition de la Palestine entre un État juif et un État arabe) et le $1^{\text {er }}$ septembre 1948 furent considérés comme des "absents " (nifkadim). Par ailleurs, ceux qui avaient fui pendant les hostilités et qui revinrent ensuite furent considérés comme des "présents absents » (nokhahim nifkadim) qui perdirent également leurs droits de propriété.

44. «Die Araber (...), die hauten ab, denn inzwischen kam ja der State of Israel, der wurde ja geschaffen, nicht ? Die Araber zogen alle ab und kamen nicht mehr zurück. (...) Ich erwähne die Drusen, weil die Drusen nicht weggelaufen sind. Und die sind nicht beteiligt gewesen und die hat man immer vom Sicherheitsstandpunkt aus als gleichwertig mit den Juden betrachtet. (...) Ich hasse die Araber nicht, im Gegenteil, ich bin der Ansicht, wir müssen zusammenleben. (...) Und wir sind ein Furunkel, sind ein Schmerz im Mittleren Osten. Die Araber verstehen nur zwei Sprachen, die eine ist Arabisch, ja, und die andere, dass der Gegner stärker ist als er. Andere Sprachen können wir mit ihnen nicht reden. (...) Wir passen hier nicht in die Mentalität rein. Wir sind keine Orientalen. Ich spreche hier nicht über die paar Juden, die da in Nordafrika früher gelebt haben oder irgendwo mit den Arabern zusammen. Die Mehrheit kommt aus anderen Ländern und wir sind nicht bereit, die arabischen Lebensgewohnheiten anzunehmen. Und infolgedessen passt unsere Kultur, unsere Weltanschauung hier nicht rein in den Mittleren Osten. » IC1, Entretien Anne Betten avec Ernst Georg Martin Pfeffermann, Haïfa, 01/05/1991.

45. « Ich habe mich etwas extrem ausgedrückt mit dem Furunkel im Mittleren Osten. » Ibid. 
46. Voir Ella Shohat, "Sephardim in Israel : Zionism from the Standpoint of ist Jewish Victims ", in Anne McClintock, Aamir Mufti \& Ella Shohat (éds.), Dangerous Liaisons: Gender, Nation, and Postcolonial Perspectives, Minneapolis, 1998, p. 39-68.

47. Ces stigmates rejoignent le soupçon de saleté associé à l'image antisémite des juifs à peau foncée dans l'Europe des XVIII ${ }^{\mathrm{e}}$ et XIX ${ }^{\mathrm{e}}$ siècles. Voir notamment Sander L. Gilman, On Blackness without Blacks : Essays on the Image of the Black in Germany, Boston, 1982.

48. "[Zionism] can be read as an extreme expression of the desire to assimilate Jews into the Western narrative of enlightenment and redemption. (...) Zionists developed a range of attitudes toward the Orient and toward the Arabs, from romantic desire to a total denial; but all of them remained within the framework of orientalist dichotomy, and served to create the 'new Jew', whom Zionism wished to define as a new European, and not an oriental. In this process, the same terms employed against the Jews in Europe (...) were adopted in order to reshape the Jewish collectivity as a national one, and were applied, albeit in different ways, to the Palestinians and the Jews from Arab countries. Orientalism is intrinsic to all the categorical dichotomies commonly used to describe Israeli society: Jews/Arabs, Ashkenazim/Mizrahim, as well as secular/religious." Amnon Raz-Krakotzkin: "The Zionist Return to the West and the Mizrahi Jewish Perspective », in Ivan Davidson Kalmar \& Derek J. Penslar (éds.), Orientalism and the Jews, Waltham (Mass.), 2005, p. 166.

\section{ABSTRACTS}

L'expérience de la migration forcée est éminemment spatiale. Elle inclut un voire plusieurs déplacement(s) - déplacement des corps dans l'espace, déplacement des repères et du cadre socio-culturels. L'espace de l'exil et de la migration est donc un espace construit où interagissent, notamment, les représentations héritées de «là-bas » et les représentations acquises « ici ». Les narrations produites par l'expérience d'une vie dans la post-migration (histoire orale) gagnent être (re)lues sous l'angle de la spatialité. Le présent article interroge la manière dont un espace d'exil se constitue par la narration. L'espace vécu (erlebter/erfahrener Raum) par les personnes contraintes de fuir l'Allemagne nazie a été profondément bouleversé par la migration forcée - a fortiori lorsqu'elles se sont retrouvées en Erets Israël, lieu mythique de l'aliya mais aussi espace largement inconnu et étranger.

Die Zwangsmigration ist auch eine räumliche Erfahrung, in der verschiedene EntOrtungsphänomene stattfinden: räumliche Ent-Ortung der Körper, Ent-Ortung der soziokulturellen Referenzrahmen. Der «Ort » des Exils und der Migration ist also ein äußerst konstruierter Raum, in dem Vorstellungen von «hier» und «da» miteinander verknüpft werden. Die in der Post-Migration produzierten Lebenserzählungen (Oral History) stellen eine geeignete Quelle dar, um räumliche Erzählstrategien zu verdeutlichen. Im vorliegenden Artikel wird die narrative Konstruiertheit eines Exil- und Migrationsraumes untersucht. Der erlebte / erfahrene Raum der nach Palästina/Erez Israel ausgewanderten Juden aus Deutschland wurde vom Migrationsprozess grundlegend verändert. Erez Israel war zugleich der mythische Ort der Alija sowie ein unbekannter und fremder Raum. 
INDEX

Geographical index: Allemagne, Palestine, Israël

Mots-clés: Juifs allemands, yekkes, histoire orale, genre, masculinité

Chronological index: $1933-1945$, post-1945

\section{AUTHOR \\ PATRICK FARGES}

Patrick Farges est Maître de conférences au Département d'Etudes germaniques de la Sorbonne Nouvelle. Sa recherche actuelle porte sur une histoire des masculinités germano-juives dans un contexte de rupture biographique. Il est l'auteur de Le trait d'union ou l'intégration sans l'oubli. Itinéraires d'exilés germanophones au Canada après 1933 Paris, éd. de la MSH, 2008) et co-auteur de Le premier féminisme allemand 1848-1933. Un mouvement social de dimension internationale (Villeneuve d'Ascq, Presses du Septentrion, 2012). 